\section{Cause of respiratory paralysis: kidney, not the lung}

\author{
Laxmikant Ramkumarsingh Tomar, Mukul \\ P. Agarwal, Surender Rajpal, Amitesh \\ Aggarwal
}

Department of Medicine, University
College of Medical Sciences
(University of Delhi), Delhi, India

\begin{abstract}
Hypokalemia is a common electrolyte abnormality and if neglected, it may be fatal. This type of electrolyte abnormality is seen in distal renal tubular acidosis (RTA). Respiratory paralysis in distal RTA due to hyokalemia is a rare clinical entity described in the literature. Here we discuss one such case: a 25 -year-old female presented with complaints of acute onset symmetrical weakness of bilateral lower limbs that rapidly progressed to involve trunk, upper limb and neck. Later on, she developed respiratory paralysis and was put on mechanical ventilation. Diagnosis of distal RTA with hypokalemia was made and managed successfully.
\end{abstract}

\section{Introduction}

Distal renal tubular acidosis (RTA) is a rare renal disorder, characterized by normal anion gap, hyperchloremic acidosis and hypokalemia, and failure to secrete acid into the urine. Respiratory paralysis due to distal RTA is an even rarer phenomenon and should be distinguished from familial periodic paralysis (FPP), as management of disease can increase the severity of the other.

\section{Case Report}

A 25-year-old female presented with acute onset weakness of both lower limbs for eight hours prior to admission. Onset of weakness started with both lower limbs within half an hour of getting up in the morning. It rapidly progressed to involve trunk, upper limbs and neck in the matter of six to eight hours. Weakness was symmetrical in pattern, first involved distal muscles, later on proximal ones and associated with heaviness in calf prior to onset of weakness. She had no history of fever, vomiting or diarrhea. There was no symptom or signs suggestive of hyperthyroidism or hypothyroidism. Patient denied any history of heavy meal intake, exposure to cold or heavy exercise prior to this weakness. No past history of dry eyes, dry mouth, dry skin, rash, photosensitivity, alopecia, jaundice, blood transfusion could be elicited. There was no history of hypertension, diabetes mellitus or past exposure to chemicals and toxins or any drug intake. No history of similar weakness previously was elicited. Family history was unremarkable.

At the time of presentation, she was conscious, oriented. She was afebrile, pulse rate was $86 / \mathrm{min}$ and blood pressure was $113 / 72 \mathrm{~mm}$ Hg. Neurological examination showed flaccid limbs (power 0/5) with decreased tone in all limbs. The deep tendon reflexes and neck holding were absent. Rest of systemic examination was normal. In the evening, the patient was found to have developed heaviness and drooping of both eyelids without involvement of extraocular muscles and pupillary sparing, followed by labored breathing and was found to be using accessory muscles of respiration. Her single breath count was 10 . She was intubated and mechanically ventilated.

Routine blood group chemistry revealed hemoglobin $9.7 \mathrm{~g} / \mathrm{dL}$, total leukocyte count $11,400 / \mathrm{mm}^{3}$, blood urea $39 \mathrm{mg} / \mathrm{dL}$, serum cretanine $1 \mathrm{mg} / \mathrm{dL}$, serum sodium $145 \mathrm{mEq} / \mathrm{L}$, serum potassium $1.6 \mathrm{mEq} / \mathrm{L}$, blood sugar 84 $\mathrm{mg} / \mathrm{dL}$, corrected serum calcium $7.3 \mathrm{mg} / \mathrm{dL}$, phosphorus $3.1 \mathrm{mg} / \mathrm{dL}$, serum albumin $3.1 \mathrm{~g} / \mathrm{dL}$, intact parathyroid hormone $78 \mathrm{pg} / \mathrm{mL}$ (normal range: $7-65 \mathrm{pg} / \mathrm{mL}$ ) and vitamin D levels were within normal limits. Her arterial blood gas analysis (ABG) pH 6.99, $\mathrm{HCO3}^{-} 15.3 \mathrm{mE} / \mathrm{L}$, $\mathrm{pCO}_{2} 67 \mathrm{~mm} \mathrm{Hg}$, chloride $108 \mathrm{mEq} / \mathrm{L}$, and anion gap 9. Urinary examination revealed $\mathrm{pH} 7.8$ and positive urinary anion gap. Antinuclear antibodies (ANA), HbSAg, anti HCV, thyroid profile, anti SS-A \& anti SS-B tests were negative. There were hypokalemic changes, $\mathrm{U}$ waves and ST depression in electrocardiogram (ECG) (Figure 1). X ray kidney ureter bladder (KUB) showed bilateral renal calculi (Figure 2). Abdominal sonography showed normal size kidney with bilateral renal calculi.

In the view of the above findings of acute flaccid quadriparesis, metabolic acidosis, hypokalemia, hypocalcemia, alkaline urine, a diagnosis of distal (type I) renal tubular acidosis leading to hypokalemic paralysis was made. Patient was managed on intravenous potassium chloride in $20 \%$ mannitol, syrup potassium citrate and oral sodium bicarbonate. Her ECG changes were reverted (Figure 3). After being mechanically ventilated for two days, she made unprecedented recovery in terms of recovery of motor weakness, in the order of: diaphragm, ocular muscles causing ptosis, distal lower limb, neck, upper limb, trunk, proximal lower extremity.
Correspondence: Laxmikant Ramkumarsingh Tomar, Department of Medicine, University College of Medical Sciences (University of Delhi), Dilshad Garden, 110095 Delhi, India. Tel: +91.9650082899 .

E-mail: drlaxmikantucms@yahoo.com

Key words: Renal tubular acidosis; Hypokalemia; Respiratory paralysis.

Contributions: LRT, total workup of patient done while patient was admitted and during her follow up; MPA, final draft of paper and help in management of patient; SR, help in management of patient; AA, contribution in writing of the manuscript; all the authors have seen the final manuscript and approved it for submission.

Conflict of interest: the authors declare no potential conflict of interest.

Received for publication: 23 November 2014.

Revision received: 16 January 2015.

Accepted for publication: 19 January 2015.

This work is licensed under a Creative Commons Attribution 3.0 License (by-nc 3.0).

(C) Copyright L.R.Tomar et al., 2015

Licensee PAGEPress, Italy

Emergency Care Journal 2015; 11:4837

doi:10.4081/ecj.2015.4837

\section{Discussion}

Distal RTA is a disorder of tubular acid transport. It is characterized by a normal anion gap hyperchloremic metabolic acidosis, with hypokalemia and failure to acidify urine below 5.5 despite systemic acidosis. It differs from uremic acidosis which is associated with a high anion gap.

Kalita and colleagues showed that out of 52 patients with hypokalemic periodic paralysis, only seven (13.5\%) had distal RTA. ${ }^{1}$ Only few cases are reported in the literature that showed respiratory paralysis due to hypokalemia in distal RTA. Distal RTA is of two types: inherited or acquired. Autosomal-recessive form presents in infancy, whereas autosomal-dominant form may not present until young adulthood. ${ }^{2}$ Acquired forms of distal RTA may be due to drugs like amphotericin B, toluene toxicity, infections like pyelonephritis or hepatitis and autoimmune diseases like Sjogrens syndrome or lupus. ${ }^{3,4}$ It often presents as hypercalciuria, nephrocalcinosis, rickets or osteomalacia, and growth retardation in children with progress to short stature in adult life. During stressful condition or fever in RTA, acidosis and hypokalemia may be life threatening. Ventricular fibrillation and even nocturnal death have been reported with RTA. ${ }^{5}$ 


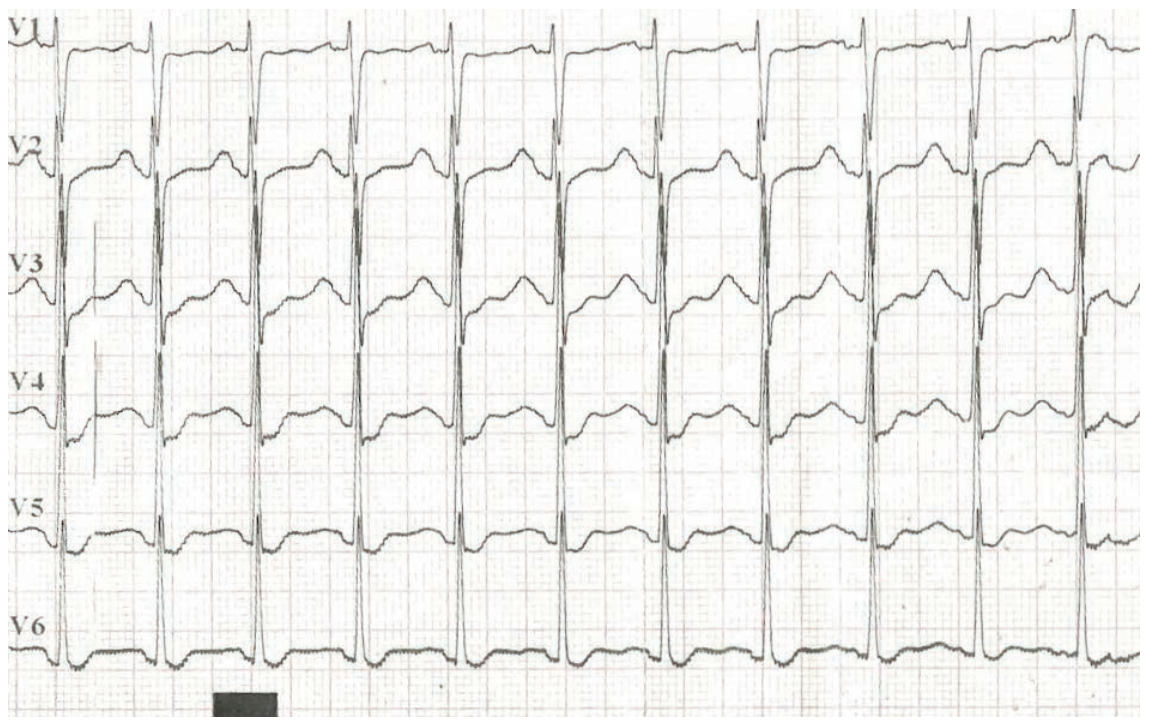

Figure 1. Electrocardiogram showed ST depression and $U$ waves suggestive of hypokalemic changes.

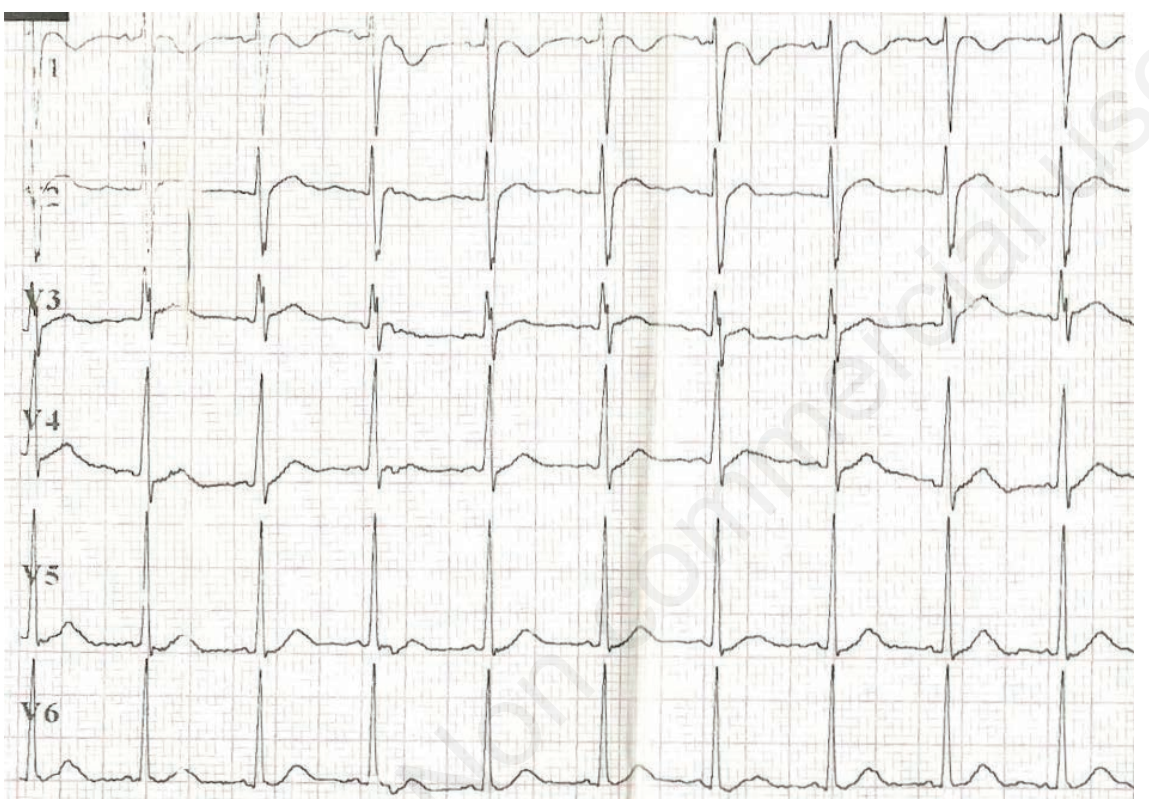

Figure 3. Electrocardiogram changes reverted after correction of hypokalemia.

Inhibition/dysfunction of certain ion exchangers and certain antibodies in distal renal tubular cells causes less $\mathrm{H}^{+}$secretion in distal renal tubular cells and leads to metabolic acidosis: ${ }^{6-8}$ absence or nonfunctioning $\mathrm{H}^{+}$ ATPase in the apical part of renal tubule causes decrease of $\mathrm{H}^{+}$excreation from intercalated cells; deficiency or malfunctioning of cytoplasmic anion-exchanger 1 (HCO3/Cl-ATPase) in the basal membrane prevents $\mathrm{HCO} 3$ resorption and less $\mathrm{H}^{+}$formation and excretion; higher antibody titres against carbon anhydrase II diminishing $\mathrm{H}$ production for secretion.

The electronegative gradient that was gen- erated by distal sodium resorption is therefore compensated by enhanced $\mathrm{K}$ excretion in the principal cells in order to balance electronegativity. Also, the metabolic acidosis can lead to less sodium absorption in the proximal tubule due to low glomerular filtrated bicarbonate that creates a hyperreninaemic hyperaldosteronism state ultimately leads to distal sodium reabsorption and potassium wasting.

The diagnosis was mainly made by serum electrolytes, ABG, urinary $\mathrm{pH}, 24$-hour urine citrate and calcium, ECG and USG. In general, if the test is non-conclusive an acid load test can often be performed. The mainstay of treat-

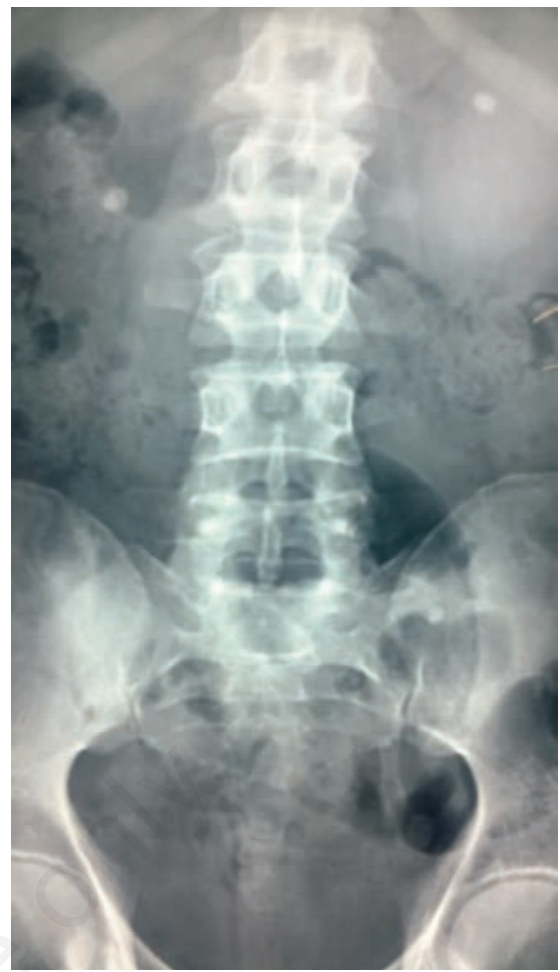

Figure 2. $\mathrm{X}$ ray abdomen showed bilateral renal calculi.

ment for distal RTA is to replenish potassium and bicarbonate levels with intravenous and oral potassium chloride or potassium citrate and oral sodium bicarbonate respectively.

Close differential diagnosis is FPP. It should be distinguished clinically by history and also on the basis of ABG. The onset in FPP is before the age of 16 , with history of episodic flaccid weakness and these episodes are precipitated by carbohydrate-rich meals, emotional or physical stress, alcohol ingestion, and cold exposure. There is rarely involvement of ocular, bulbar, and respiratory muscles. ${ }^{9}$ There is redistribution of $\mathrm{K}^{+}$across the cell membrane in skeleton muscle due to mutation in two genes i.e. voltage-gated calcium or sodium channel (CACNA1S and SCN4A).$^{10}$ Therefore, administration of sodium bicarbonate in FPP causes intracellular shift of potassium into cells and further produces hypokalemia. Acetazolamide and oral potassium supplement is the treatment modality for FPP.

Generally, correction of the serum $\mathrm{pH}$ and bicarbonate levels reduces renal potassium wasting by diminishing distal sodium delivery, but sometimes mild hypokalaemic state can persist and can be corrected by simultaneous potassium supplementation. Administration of acetazolamide in distal RTA is contraindicated as it produces more hypokalemia. 


\section{Conclusions}

Every patient with hyperchloremic metabolic acidosis associated with unexplained hypokalemia should be assessed for RTA, as hypokalemia may cause quadriparesis or it may end up in a life threatening complication like respiratory paralysis. This should also be differentiated from FPP especially during acute attack, as management of one disease increases the severity of the other.

\section{References}

1. Kalita J, Goyal G, Bhoi SK, et al. Comparative study of thyrotoxic periodic paralysis from idiopathic hypokalemic periodic paralysis: an experience from
India. Ann Indian Acad Neur 2012;15:18690 .

2. Karet FE. Inherited distal renal tubular acidosis. J Am Soc Nephrol 2002;13:217884.

3. McCurdy DK, Frederic M, Elkinton JR. Renal tubular acidosis due to amphotericin B. New Engl J Med 1968;278:12430 .

4. Carlisle EJ, Donnelly SM, Vasuvattakul S, et al. Glue-sniffing and distal renal tubular acidosis: sticking to the facts. J Am Soc Nephrol 1991;1:1019-27.

5. Brenner BM. The kidney. 7th ed. Philadelphia, PA, USA: Saunders; 2004.

6. Christensen KS. Hypokalemic periodic paralysis secondary to renal tubular acidosis. Eur Neurol 1985;24;303-5.

7. Walsh S, Turner CM, Toye A, et al. Immunohistochemical comparison of a case of inherited distal renal tubular aci- dosis (with a unique AE1 mutation) with an acquired case secondary to autoimmune disease. Nephrol Dial Transplant 2007;22:807-12.

8. Han JS, Kim GH, Kim J, et al. Secretorydefect distal renal tubular acidosis is associated with transporter defect in $\mathrm{H}(+)$ ATPase and anion exchanger-1. J Am Soc Nephrol 2002;13:1425-32.

9. Takemoto F, Hoshino J, Sawa N, et al. Autoantibodies against carbonic anhydrase II are increased in renal tubular acidosis associated with Sjogren syndrome. Am J Med 2005;118:181-4.

10. Sternberg D, Maisonobe T, Jurkat-Rott K, et al. Hypokalaemic periodic paralysis type 2 caused by mutations at codon 672 in the muscle sodium channel gene SCN4A. Brain 2001;124:1091-9. 